\title{
A HELLY TYPE THEOREM ON THE SPHERE
}

\author{
MEIR KATCHALSKI
}

Abstract. This paper establishes a Helly type theorem for convex sets in $S_{n}$, the $n$-dimensional unit sphere.

1. Introduction. Let $S_{n}$ denote the $n$-dimensional unit sphere in Euclidean $(n+1)$-dimensional space, $R^{n+1}$.

A subset of $S_{n}$ will be called convex if it is the intersection of $S_{n}$ with a convex cone with apex 0 in $R^{n+1}$.

A subset of $S_{n}$ will be called strongly convex if it is convex and does not contain antipodal points.

Thus a set consisting of two antipodal points is convex but not strongly convex.

For other definitions of convex sets in $S_{n}$ and for a survey of Helly type theorems see [2]. For standard notation and terminology see [3].

M. J. C. Baker has proven in [1] the following

THEOREM. For all positive integers $n$ and $t$, if the intersection of each $n+1$ members of a family of at least $n+1+2 t$ strongly convex sets in $S_{n}$ is nonempty then the intersection of some $n+1+t$ members of the family is nonempty.

The purpose of this paper is to generalize Baker's result by proving

THEOREM A. Let $n$ and $t$ be positive integers and let $\mathbb{Q}$ be $a$ family of $n+1+t$ convex sets in $S_{n}$.

If every $n+1$ members of $\mathbb{Q}$ have nonempty intersection then the intersection of some $n+1+[t / 2]$ members of $\mathbb{Q}$ is nonempty.

Moreover, $n+1+[t / 2]$ cannot be replaced by $n+[t / 2]+2$.

It is possible to improve Theorem A for strongly convex sets and $t=2$ by establishing

THEOREM B. Let $n$ be an integer and let $\mathbb{Q}$ be a family of $n+3$ strongly convex sets in $S_{n}$. Suppose that every $n+1$ members of $\mathbb{Q}$ have nonempty intersection.

If $k$ is the number of different $n+2$ membered subfamilies $\mathscr{B}$ of $Q$ such that $\cap \Re=\varnothing$ then $k \leqslant 2$.

Received by the editors October 14, 1976.

AMS (MOS) subject classifications (1970). Primary 52A20, 52A35.

Key words and phrases. Convex sets, $n$-dimensional unit sphere, Helly type theorems.

C American Mathematical Society 1977 
The proof of Theorem $A$ is based on

(1) Properties of certain families of convex cones called nondegenerate families (N.D.F.'s), discussed in [4] and [5].

(2) The existence, or the nonexistence of certain $k$-neighborly polytopes in $R^{n}$; see [3].

All the necessary definitions and properties of N.D.F.'s and of neighborly polytopes are stated in $\$ 2$.

A proof of Theorem $A$ is given in $\$ 3$.

The proof of Theorem B is similar to the proof of Theorem A and is therefore omitted.

ACKNowledgement. The author wishes to express his thanks to Professor Grünbaum for his help and advice.

2. Nondegenerate families and neighborly polytopes. Let $\mathcal{Q}$ be a nonempty finite family of convex cones with apex 0 in $R^{n}$.

The family $\mathbb{Q}$ will be called a nondegenerate family (a N.D.F.) if each member of the family is of dimension $n$, the intersection of any two members of the family is at least of dimension $(n-1), \ldots$, the intersection of any $n$-members is at least of dimension 1 and the intersection of all members is $\{0\}$.

A subset $\mathscr{B}$ of $\mathbb{Q}$ will be called a face of $Q$ if $\cap(Q \backslash \mathscr{B})$ is a subspace.

A subset $\mathscr{B}$ of $\mathbb{Q}$ is a $k$-face of $\mathscr{Q}$ if it is a face of $\mathscr{Q}$ and if $\operatorname{dim} \cap(\mathbb{Q} \backslash \mathscr{B})$ $=|\mathscr{B}|-k \cdot(|\mathscr{B}|$ means the cardinality of $\mathscr{B}$.

A relationship between N.D.F.'s and polytopes is given by

THEOREM C. (i) If $\mathbb{Q}$ is a N.D.F. in $R^{n}$ and $|\mathbb{Q}|=n+t$ then there exists $a$ $(t-1)$-polytope $P$ in $R^{t-1}$ such that

(1) The lattice of faces of $P$ and the lattice of faces of $\mathbb{Q}$, both ordered by the inclusion relation, are isomorphic. The isomorphism carries $k$-faces of $P$ to $(k+1)$-faces of $Q$.

(ii) For any $(t-1)$-polytope $P$ with $n+t$ vertices there exists a N.D.F. Q in $R^{n}$ with $|\mathbb{Q}|=n+t$ such that (1) is satisfied.

A proof of Theorem $\mathrm{C}$ is given in [5].

NeIGHBORLY POLYTOPES. A polytope $P$ is $k$-neighborly if every subset of $k$ vertices of $P$ is the set of vertices of a proper face of $P$.

Proofs of the following two theorems may be found in Chapter 7 of [3].

THEOREM D. If $P$ is a $k$-neighborly $d$-polytope then:

(i) All $k$ vertices of $P$ are affinely independent and $P$ is $k^{\prime}$ neighborly for $1<k^{\prime}<k$.

(ii) if $k>\left[\frac{1}{2} d\right]$ then $p$ is a simplex.

THEOREM E. For every $d$ and $v>d$ there exist $d$-polytopes with vertices which are $\left[\frac{1}{2} d\right]$ neighborly. 
3. Proof of Theorem A. In order to prove Theorem A it is sufficient to prove

THEOREM F. Let $n$ and $t$ be nonnegative integers. Let $\mathbb{Q}$ be a family of $n+t$ convex cones with apex 0 in $R^{n}$ such that

$$
\cap \mathbb{Q}=\{0\}
$$

and

$$
\cap \mathscr{B} \neq\{0\} \text { for any } n \text { membered subfamily } \mathscr{B} \text { of } \mathcal{Q} .
$$

Then there exists an $n+[t / 2]$ membered subfamily $\mathcal{C}$ of $\mathbb{Q}$ such that $\cap \mathcal{C} \neq\{0\}$.

Moreover, $n+[t / 2]$ in the last statement cannot be replaced by $n+[t / 2]+$ 1.

Proof of Theorem F. The proof is by induction on $n$. The cases $n=0$ and $t \leqslant 1$ are trivial, so assume that $n>0$ and that $t \geqslant 2$.

Let $\mathscr{Q}$ be a family of convex cones with apex 0 on $R^{n}$ such that $|\mathfrak{Q}|=n+$ $t$ and assume that (2) and (3) are satisfied.

Suppose that $A$ is not a N.D.F. let $\mathscr{N}$ be a maximal subset of $\mathscr{Q}$ such that

$$
n^{\prime}=\operatorname{dim} \cap \Re \leqslant n-|\Re| \text {. }
$$

Clearly $\phi$ satisfies (4) since $\operatorname{dim} \cap \phi=n=n-|\phi|$.

It is not difficult to verify that $\operatorname{dim} \cap \Re=n-|\Re|$ and that $\Re \neq \phi$ since $\mathcal{Q}$ is not a N.D.F.

Define a family $\mathbb{Q}^{\prime}=\{\cap \mathfrak{R} \cap\{A\}: A \in \mathbb{Q} \backslash \mathfrak{R}\}$ of convex cones with apex 0 in $R^{\prime}=\operatorname{span} \cap \Re$.

Since $\left|\mathbb{Q}^{\prime}\right|=n+t-|\Re|=n^{\prime}+t$ and since $\mathbb{Q}^{\prime}$ satisfies (2) and (3) we can use the induction hypothesis to obtain a $\mathcal{C}^{\prime} \subset \mathbb{Q} \backslash \mathfrak{R}$ such that $\left|\mathcal{C}^{\prime}\right| \geqslant n^{\prime}$ $+[t / 2]$ and $\cap \mathcal{C}^{\prime} \neq\{0\}$.

Define $C=C^{\prime} \cup \Re$. The subfamily $\mathcal{C}$ has the desired properties since

$$
|\mathfrak{C}|=\left|\mathfrak{C}^{\prime}\right|+|\mathfrak{T}| \geqslant n^{\prime}+[t / 2]+|\mathfrak{T}|=n+[t / 2]
$$

and

$$
\cap \mathcal{C}=\cap \mathcal{C}^{\prime} \neq\{0\}
$$

Suppose that $A$ is a N.D.F. Assume by contradiction that $\cap \mathscr{B}=\{0\}$ for any $n+[t / 2]$ membered subfamily $\mathscr{B}$ of $\mathcal{Q}$. Thus for any $\mathcal{C} \subset \mathbb{Q}$ such that

$$
|\mathfrak{C}| \leqslant n+t-(n+[t / 2])=t-[t / 2],
$$

$\mathcal{C}$ is a $|\mathcal{C}|$-face of $\mathcal{Q}$.

Let $P$ be the $(t-1)$-polytope described in Theorem C. It follows from (1) of Theorem $\mathrm{C}$ that $P$ is a $t-[t / 2]$ neighborholy polytope with $n+t$ vertices. Since $t-[t / 2]>[(t-1) / 2], P$ is by Theorem $\mathrm{D}$ a $(t-1)$-simplex and therefore $n+t=(t-1)+1$, so that $n=0$, a contradiction. This completes the proof of the first part of Theorem $F$.

To complete the proof of Theorem $\mathrm{F}$ let $P$ be a $[(t-1) / 2]$ neighborly $(t-1)$-polytope with $n+t$ vertices (see Theorem E). 
Let $\mathcal{Q}$ be the N.D.F. described in (ii) of Theorem C. The family satisfies (2) and (3) since any N.D.F. in $R^{n}$ satisfies (2) and also (3) if $n>0$.

For any $\mathscr{B} \subset \mathbb{Q}$ such that $\mathscr{B} \geqslant n+[t / 2]+1$ we have that

$$
|Q \backslash \mathscr{B}| \leqslant t-[t / 2]-1 \leqslant[(t-1) / 2] \text {. }
$$

By Theorem $C(i)$, for any such $\mathscr{B}, \mathbb{Q} \backslash \mathscr{B}$ is a $|Q \backslash \mathscr{B}|$-face of $\mathcal{Q}$.

Therefore $\operatorname{dim} \cap \mathscr{B}=\operatorname{dim} \cap(\mathbb{Q} \backslash(\mathbb{Q} \backslash \mathscr{B}))=|\mathbb{Q} \backslash \mathscr{B}|-|\mathbb{Q} \backslash \mathscr{B}|=0$ so that $\cap \mathscr{B}=\{0\}$. The proof of Theorem $F$ is now complete.

\section{REFERENCES}

1. M. J. C. Baker, A spherical Helly type theorem, Pacific J. Math. 23 (1967), 1-3. MR 36 \#5820.

2. L. Danzer, B. Grünbaum and V. Klee, Helly's theorem and its relatives, Proc. Sympos. Pure Math., vol. 7, Amer. Math. Soc., Providence, R. I., 1963, pp. 101-180. MR 28 \# 524.

3. B. Grünbaum, Convex polytopes, Interscience, New York, 1967. MR 37 \#2085.

4. M. Katchalski, Some properties of families of convex cones, Trans. Amer. Math. Soc. (to appear).

5. __ Non-degenerate families of convex cones and convex polytopes, Discrete Math. (to appear).

Department of Mathematics, University of Alberta, Edmonton, Alberta T6G 2Gl, CANADA 